\title{
BMJ Open Intravenous high-dose vitamin $C$ for the treatment of severe COVID-19: study protocol for a multicentre randomised controlled trial
}

\author{
Fang Liu, Yuan Zhu, Jing Zhang, Yiming Li, Zhiyong Peng (D)
}

To cite: Liu F, Zhu Y, Zhang J, et al. Intravenous high-dose vitamin $\mathrm{C}$ for the treatment of severe COVID-19: study protocol for a multicentre randomised controlled trial. BMJ Open 2020;10:e039519. doi:10.1136/ bmjopen-2020-039519

- Prepublication history for this paper is available online. To view these files, please visit the journal online (http://dx.doi. org/10.1136/bmjopen-2020039519).

FL and YZ contributed equally.

Received 21 April 2020

Revised 23 June 2020

Accepted 25 June 2020

Check for updates

(C) Author(s) (or their employer(s)) 2020. Re-use permitted under CC BY-NC. No commercial re-use. See rights and permissions. Published by BMJ.

Department of Critical Care Medicine, Zhongnan Hospital of Wuhan University, Wuhan, Hubei, China

Correspondence to

Dr Zhiyong Peng;

Pengzy5@hotmail.com

\section{ABSTRACT}

Introduction The rapid worldwide spread of COVID-19 has caused a global health crisis. To date, symptomatic supportive care has been the most common treatment. It has been reported that the mechanism of COVID-19 is related to cytokine storms and subsequent immunogenic damage, especially damage to the endothelium and alveolar membrane. Vitamin C (VC), also known as Lascorbic acid, has been shown to have antimicrobial and immunomodulatory properties. A high dose of intravenous VC (HIVC) was proven to block several key components of cytokine storms, and HIVC showed safety and varying degrees of efficacy in clinical trials conducted on patients with bacterial-induced sepsis and acute respiratory distress syndrome (ARDS). Therefore, we hypothesise that HIVC could be added to the treatment of ARDS and multiorgan dysfunction related to COVID-19.

Methods and analysis The investigators designed a multicentre prospective randomised placebo-controlled trial that is planned to recruit 308 adults diagnosed with COVID-19 and transferred into the intensive care unit. Participants will randomly receive HIVC diluted in sterile water or placebo for 7 days once enrolled. Patients with a history of VC allergy, end-stage pulmonary disease, advanced malignancy or glucose-6-phosphate dehydrogenase deficiency will be excluded. The primary outcome is ventilation-free days within 28 observational days. This is one of the first clinical trials applying HIVC to treat COVID-19, and it will provide credible efficacy and safety data. We predict that HIVC could suppress cytokine storms caused by COVID-19, help improve pulmonary function and reduce the risk of ARDS of COVID-19.

Ethics and dissemination The study protocol was approved by the Ethics Committee of Zhongnan Hospital of Wuhan University (identifiers: Clinical Ethical Approval No. 2020001). Findings of the trial will be disseminated through peer-reviewed journals and scientific conferences. Trial registration number NCT04264533.

\section{INTRODUCTION}

\section{Background and rationale}

The COVID-19 pandemic is a threat that has caused panic at the global level. As of 21 June 2020, 8708008 cases were confirmed worldwide, resulting in 461715 deaths. ${ }^{1}$ Infected patients presented predominantly with fever

\section{Strengths and limitations of this study}

- This is one of the first prospective randomised controlled trials applying high dose of intravenous vita$\min$ C (HIVC) to treat COVID-19.

- 'High-dose' vitamin C therapy lacks a universal definition. A previous meta-analysis considered high doses as equal to or greater than $10 \mathrm{~g} /$ day. In this trial, we will administer $24 \mathrm{~g}$ vitamin $\mathrm{C}$ per day for 7 days intravenously.

- HIVC has advantages in terms of stability, availability, safety and cost compared with other treatments.

- The sample size was calculated in two stages to ensure the calculation is reasonable, maximises the possibility of obtaining significant results and provides credible outcome data.

- As the duration and distribution of infected cases are unpredictable geographically and temporally, the number of recruited patients at each centre is also unpredictable, in spite of competitive enrolment.

and cough as well as dyspnoea and myalgia. ${ }^{2-4}$ A meta-analysis ${ }^{4}$ concluded that, among the patients, $32.8 \%$ presented with acute respiratory distress syndrome (ARDS), 20.3\% were transferred to the intensive care unit (ICU) and $13.9 \%$ died. According to our previous research, ${ }^{3}$ patients with ARDS accounted for $66.1 \%$ of patients with COVID-19 in the ICU, and the rates of non-invasive and invasive mechanical ventilation were $41.7 \%$ and $47.22 \%$, respectively. However, no specific treatment is currently available because traditional antiviral drugs do not work well against COVID-19. Recent clinical trials exploring new therapies, including remdesivir, $^{5}$ hydroxychloroquine ${ }^{6}$ and lopinavirritonavir, ${ }^{7}$ for COVID-19 had negative results. Therefore, it is urgent to explore effective therapies considering the grim situation.

The mechanism of COVID-19 involves a cytokine storm, which is a potentially fatal immune reaction triggered by a variety of factors, including infections. Cytokine storms 
are associated with the clinical manifestation of severe inflammation and highly elevated levels of proinflammatory cytokines. ${ }^{8}$ Previous research ${ }^{9}$ suggested that cytokine storms may be the main mechanism of highly pathogenic human coronavirus infected pneumonia, such as severe acute respiratory syndrome and Middle East respiratory syndrome. As a member of the coronavirus family, ${ }^{10}$ severe acute respiratory syndrome coronavirus 2 (SARS-CoV-2) exhibits similar clinical features. The clinical characteristics of COVID-19 indicate that cytokine storms may be positively correlated with the severity of the disease. ${ }^{211} 12$ Moreover, cytokines and T cell subsets may be indicators for predicting prognosis. ${ }^{12} \mathrm{An}$ immunopathology report ${ }^{12}$ speculated that interleukin (IL)-6 and granulocyte-macrophage colony-stimulating factor (GM-CSF) were the main cytokines in the hyperinflammatory response caused by COVID-19, while Th1 cells were the key cells involved, especially for patients transferred into ICUs. The findings and speculations were verified by an autopsy study that confirmed that patients with critically ill COVID- $19^{13}$ had developed ARDS. In addition, they also found that the overactivation of $\mathrm{T}$ cells and the decrease in the cell counts may explain the occurrence of cytokine storms and, to some degree, the severe immune-medicated injury. All these results indicated that decreasing cytokine storms and immunogenetic damage may be the main treatment option for critically ill patients with COVID-19.

Vitamin C (VC), a common and necessary nutrient, is also an antioxidant. In addition to its role in the metabolism of the human body, including energy transformation, collagen biosynthesis and repair, adrenal steroid and catecholamine production, iron absorption and so on, ${ }^{14-19}$ VC also possesses antimicrobial properties-thus reducing the risk of infections-and immunomodulatory functions, particularly in high concentrations. ${ }^{14}$ First, VC plays a crucial role in immunomodulation. It can inhibit the activation of nuclear factor kappa-B (NFkB), which is a primary proinflammatory transcription factor, and plays a pivotal role in overall immunity, including the genetic regulation of chemokines, cytokines, adhesion molecules, inflammatory mediators and apoptosis inhibitors. ${ }^{20} \mathrm{VC}$ can inhibit the production of IL-6 and tumor necrosis factor alpha (TNF- $\alpha),{ }^{21} 22$ and this effect appears to be a dose dependent. ${ }^{21} \mathrm{VC}$ can reduce GM-CSF signalling responses, ${ }^{23}$ functioning as a regulator of cytokine redox-signal transduction in host defence cells and having a possible role in controlling inflammatory responses. In addition, high-dose $\mathrm{VC}$ can regulate the proliferation and function of $\mathrm{T}$ cells, B cells and natural killer (NK) cells. ${ }^{24-27}$ This may help inhibit the progression of cytokine storms and improve the host's immunity. Second, previous studies $^{22}$ demonstrated that VC can inhibit oxidative stress, an important part of the innate immune response to viral respiratory infection ${ }^{28}{ }^{29}$ and contributes to lung injury and barrier dysfunction. ${ }^{28}$ Oxidative stress may also play a role in the mechanism of COVID-19. ${ }^{29}$ It has been reported that VC can repair oxidative damage in human bronchial epithelium by modulating reactive oxygen species (ROS) generation and inflammatory expression ${ }^{30}$ and can prevent ROS-induced lung damage. ${ }^{31}$ Third, VC can regulate alveolar fluid clearance by enhancing lung epithelial barrier function through epigenetic and transcriptional enhancement of protein channels that regulate alveolar fluid clearance. ${ }^{32}{ }^{33} \mathrm{VC}$ may help decrease the symptoms of ARDS and improve respiratory function. Fourth, VC may have antiviral effects. VC has been reported to inhibit the replication of herpes simplex virus 1 , poliovirus type 1 and influenza A virus in vitro. ${ }^{34}$

Previous research ${ }^{35}$ demonstrated that intravenous VC (IVC) can achieve a higher plasma concentration than oral VC due to losses during intestinal absorption, tissue transport and renal reabsorption. The efficacy and safety of intravenous high-dose VC (HIVC) in critically ill patients have been investigated via several clinical trials. A recent meta-analysis, ${ }^{15}$ of mostly cardiac surgery trials, revealed that VC shortened ICU length of stay and duration of mechanical ventilation in ICU patients. In addition, the effect of $\mathrm{VC}$ was significantly greater for patients with more severe illness. ${ }^{15} 36$ Regarding patients with sepsis or ARDS, a phase I trial of IVC in patients with severe sepsis ${ }^{37}$ reported that VC significantly reduced multiorgan failure scores and circulating injury biomarker levels. Notably, the effect appeared greater in the high-dose group $(200 \mathrm{mg} / \mathrm{kg})$ than in the low-dose group $(50 \mathrm{mg} / \mathrm{kg})$. However, another study conducted on patients with sepsis and $\mathrm{ARDS}^{38}$ reported no difference in the primary outcome of organ failure scores and inflammation biomarkers but found a significant reduction in 28-day mortality and long-term prognosis. This study applied $50 \mathrm{mg} / \mathrm{kg} \mathrm{VC}$ intravenously at a late stage when patients were undergoing mechanical ventilation. In addition, there was also a case report ${ }^{39}$ that administered HIVC $(200 \mathrm{mg} / \mathrm{kg})$ to successfully treat virusinduced ARDS, with patients making a rapid recovery after receiving extracorporeal membrane oxygenation and without any long-term sequelae.

A major concern regarding the use of high-dose VC is its potential side effects. Many reported side effects of high-dose VC are insignificant and rare and of little consequence. ${ }^{40}$ It was reported that high-dose VC was related to haemolysis in glucose-6-phosphate dehydrogenase (G-6-PD) deficiency, acute kidney injury (AKI) and acute oxalate nephropathy. ${ }^{41-44}$ However, adverse effects were mostly reported in a few cases and were related to too large of doses, ${ }^{41-44}$ non-standard administration ${ }^{43}$ or high-risk underlying diseases. ${ }^{44}$ Clinical trials with large sample sizes conducted on ICU patients ${ }^{156-38}$ reported few adverse events (AEs). In addition, a survey ${ }^{45}$ also indicated that other than the known complications of kidney damage and G-6-PD deficiency, HIVC appeared to be remarkably safe. For patients with haemochromatosis, G-6-PD deficiency, renal dysfunction, renal stones or oxaluria, VC should be carefully administered; adequate hydration, appropriate dilution and slow infusion rates are recommended for HIVC. ${ }^{46}$ 


\begin{tabular}{|c|c|c|c|c|c|}
\hline \multirow{3}{*}{ Time Point } & \multicolumn{5}{|c|}{ STUDY PERIOD } \\
\hline & \multirow{2}{*}{ Enrolment } & \multicolumn{3}{|c|}{ Treatment Period } & \multirow{2}{*}{\begin{tabular}{|c|} 
Follow-up \\
D28 \\
\end{tabular}} \\
\hline & & D1 & D3 & D7 & \\
\hline Informed consent & - & & & & \\
\hline Inclusion/exclusion criteria & - & & & & \\
\hline Randomisation & - & & & & \\
\hline Demographic date & - & & & & \\
\hline Vital sign & - & & & & \\
\hline Basic disease history & - & & & & \\
\hline Intervention & & & - & & \\
\hline Blood routine & & - & - & - & \\
\hline Urine routine & & - & - & - & \\
\hline Coagulation function & & - & - & - & \\
\hline Markers of myocardial injury ${ }^{1}$ & & - & - & - & \\
\hline Serum chemistry ${ }^{2}$ & & - & - & - & \\
\hline X-ray examination & & - & - & - & \\
\hline Ultrasonography & & - & - & - & \\
\hline Discharge or death records & & & & & - \\
\hline Adverse event monitoring & & & Lou & & \\
\hline
\end{tabular}

${ }^{1}$ Markers of myocardial injury include CK-MB, MYO, TnIU1tr

${ }^{2}$ Serum chemistry includes ALT, AST, TBIL, DBIL, CHOL, TG, Scr, BUN, CysC, BNP, PCT, cytokines.

Figure 1 Standard Protocol Items: Recommendations for Interventional Trials checklist. ALT, alanine aminotransferase; AST, aspartate aminotransferase; BNP, brain natriuretic peptide; BUN, blood urea nitrogen; $\mathrm{CHOL}$, cholesterol; CK$\mathrm{MB}$, creatine kinase isoenzyme-MB; cysC, cystatin C; DBIL, direct bilirubin; IL-6, interleukin-6; MYO, myoglobin; PCT, procalcitonin; SCR, serum creatinine; TG, triglyceride; TBIL, total bilirubin; TnlU1tr, high sensitive troponin I. ${ }^{1}$ Markers of myocardial injury include CK-MB, MYO, TnIU1tr. ${ }^{2}$ Serum chemistry includes ALT, AST, TBIL, DBIL, CHOL, TG, Scr, BUN, CysC, BNP, PCT, cytokines.

Thus, we hypothesise that administering HIVC at an early stage of ARDS in COVID-19 would result in better outcomes. In this trial, we will tentatively explore the safety and efficacy of HIVC used in COVID-19.

\section{OBJECTIVES}

The main goal is to investigate a new potential therapy for COVID-19 by clarifying the effect of HIVC on the prognosis of patients with COVID-19, especially on respiratory function assessed by ventilation-free days.

\section{METHODS}

This protocol was written in accordance with the Standard Protocol Items: Recommendations for Interventional Trials guidelines ${ }^{47}$ (see attached Research Checklist); the protocol is summarised in figures 1 and 2.

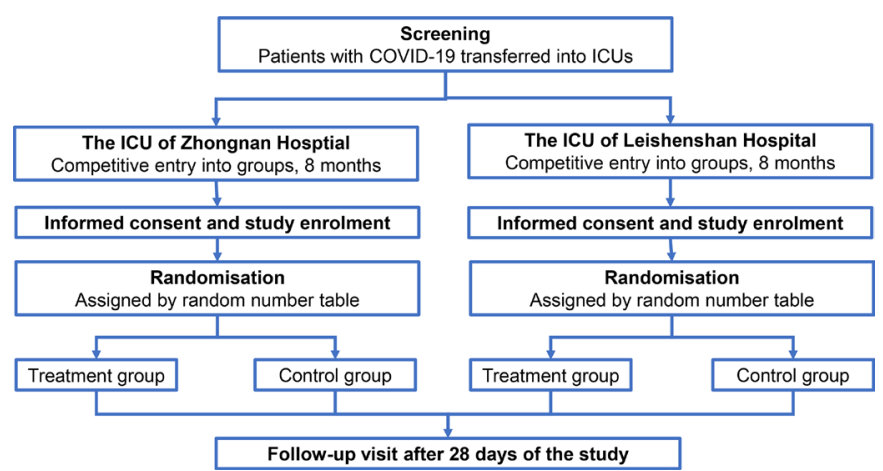

Figure 2 Study flow. ICU, intensive care unit.

\section{DESIGN AND SETTING}

The study is a multicentre, prospective randomised, placebo-controlled trial. It is an interventional study with two arms (VC group and placebo group). The study is well designed according to the concept of a pragmatic trial $^{48}$ with broad inclusion criteria, a reasonable sample size and study procedures embedded into clinical routine care and executed by clinical personnel.

\section{STUDY SITES AND PERIOD}

The trial is being conducted in the ICU of Zhongnan Hospital of Wuhan University and the ICU of Leishenshan Hospital of Wuhan City from 14 February 2020 to 30 September 2020. Zhongnan Hospital of Wuhan University, with a comprehensive 66-bed ICU, is a tertiary hospital integrating clinical care, scientific research and teaching. During the COVID-19 pandemic, it took charge of Leishenshan Hospital of Wuhan City (the second specialised hospital that was built by Wuhan government within half a month to only accommodate and treat patients with COVID-19). Each ICU will enrol patients competitively for 8 months.

\section{STUDY PARTICIPANTS}

Patients admitted into the above 2 ICUs, consistent with the following inclusion criteria and no exclusion criteria at the time of randomisation, will be considered for enrolment. Enrolment must be completed within 12 hours after patients are admitted to the ICU.

Participants' inclusion criteria are as follows:

1. Adults (age 18 years or older).

2. Diagnosed with COVID-19 according to the Diagnosis and Clinical Management of 2019 novel coronavirus infected pneumonia (trial version 5 , revised version) ${ }^{49}$

3. Having a respiratory failure index (RIF) (arterial oxygen tension (or pressure)/fractional inspired oxygen) $<300 \mathrm{~mm} \mathrm{Hg}$.

4. Being treated in the ICU.

Exclusion criteria are as follows:

1. Patients with a history of allergy to VC.

2. Patients with dyspnoea caused by cardiogenic pulmonary oedema.

3. Pregnant or lactating women. 
4. Patients with an expected survival duration of less than 24 hours.

5. Patients with a previous history of end-stage pulmonary disease, end-stage malignant tumour, G-6-PD deficiency, diabetic ketoacidosis, active kidney stone disease and severe kidney diseases.

6. Patients who are already enrolled in another clinical trial.

\section{RISKS, AES AND CONSENT}

As mentioned above, this trial is considered to be safe. First, VC is an essential nutrient for the human body, and IVC has been widely used in clinical practice for over five decades ${ }^{50}$ Second, patients with greater potential risks of side effects were excluded based on the exclusion criteria. Third, the rate of infusion is as slow as $12 \mathrm{~mL} /$ hour. However, AEs and serious adverse events (SAEs) must be observed and followed in accordance with the good clinical practice guidelines issued by the National Medical Products Administration of the People's Republic of China. ${ }^{51}$ An AE refers to any untoward medical event that occurs after a human subject receives a drug. SAEs include prolonged hospital length of stay, disability, death and so on. AEs and SAEs will be recorded during the 28 days of observation from enrolment. Either may occur during a subject's participation in the research and do not need to have a causal relationship with the treatment. Investigators will evaluate the relationship between the events and the intervention and report it to the ethical committee and data and safety monitoring board (DSMB). Benefits and potential risks are written in the informed consent document. Patients will be informed of the purpose, intervention, benefits and possible risks of the study.

\section{RANDOMISATION, ALLOCATION CONCEALMENT AND BLINDING}

Each ICU is assigned an independent, random numeric table that is generated by the primary investigator alone through Microsoft Excel 2019 and carried out by other investigators. Each table has equal numbers of 1 and 2, which represent the placebo group (sterile water for injection) and treatment group (VC), respectively. Once generated, the list will remain unchanged and be kept by the primary investigator who does not participate in the treatment of patients. When a patient is transferred to the ICU and meets the enrolment criteria, the clinician on duty will inform the other investigators and obtain a number from the list according to the chronological order of ICU recruitment. Then, participants are enrolled in the corresponding group. This trial is not completely blinded. The COVID-19 pandemic is a public health emergency that overwhelms the medical system. Thus, there is not enough manpower and material resources to implement the double-blind method, which requires modifying the medical orders system, connecting the ward and the pharmacy and assigning a special nurse to configure and label infusions. Therefore, the grouping and intervention are unknown to the participants and investigators responsible for data collection and statistical analysis but are known to the clinical staff and investigators responsible for recruiting participants. VC injection and sterile water for injection are both colourless and transparent liquids and will be contained in the same brown syringes with different marks and without explanations on the syringe to make sure that patients cannot distinguish the treatment they receive. Investigators, clinicians and DSMB will monitor the whole process of treatment and determine whether to continue the trial if potential AEs occur or if it is in the participants' best interests to stop treatment, so there is no need for unblinding of the study grouping.

\section{STUDY INTERVENTIONS}

This trial is an interventional study with two arms. The intervention lasted for 7 days. For the treatment group, $12 \mathrm{~g} \mathrm{VC}$ will be diluted in sterile water to a total volume of $50 \mathrm{~mL}$ and will be infused within 4 hours by an infusion pump. This treatment will be repeated every 12 hours. Thus, the total dosage of VC for the treatment group is $24 \mathrm{~g}$ per day. The control group is assigned a placebo $(50 \mathrm{~mL}$ sterile water); the method and speed of administration are identical to those of the treatment group. Sterile water is used for dilution fluid as well as placebo fluid, as the combination of VC and saline has been reported to irritate the vein. ${ }^{52}$ No other medications or therapeutic schedules other than standard critical supportive care will be applied in this study. The intervention should be commenced within 12 hours after enrolment. Delivery of the assigned VC injection or placebo injection is achieved through the medical orders system, pharmacy supply, and clinical routine in hospitals. VC injection is a common and basic drug and has been widely used in the hospital, so there will not be problems of inventory and logistics.

\section{DATA COLLECTION AND MANAGEMENT}

Baseline data including demographic characteristics, main diagnosis, assigned group, changes in Acute Physiology and Chronic Health Evaluation (APACHE) II scores, Sequential Organs Failure Assessment (SOFA) scores and multiorgan function, especially respiratory function indices, will be collected on the first day. General vital signs, chest radiography and CT scans, respiratory and cardiac ultrasounds, respiratory indices (RIF, parameters of ventilation and so on), infection indices (white cell count, neutrophil count, lymphocyte count, procalcitonin (PCT) and C reactive protein (CRP)), cytokine tests (IL-6, IL-10 and so on), immunological indices (lymphocyte subpopulation), organ function indices (cardiac function, hepatic function, renal function and coagulation function) and blood gas analyses will be collected on the first, third and seventh days. Participants are scheduled for a follow-up visit on the 28th day to track their long-term prognosis and sequelae. 
All the data will be collected from the clinical information system of the hospital and recorded in the case report format (CRF). Every participant will be distinguished with a study identifier and initial without full name. An electronic password-protected document containing all the information from the CRFs will be set up for statistical analysis. The analysis process will be performed by the primary investigator and designated teammates who are experienced with trial CRFs. Relevant documents will be stored at the Department of Critical Care Medicine of Zhongnan Hospital of Wuhan University after the study. The confidentiality and safety of participants' data are guaranteed. All the data will be reserved for 10 years for the purpose of further analysis and investigation.

\section{STUDY OUTCOMES}

The primary outcome of this study is ventilator-free days in the 28 days since admission to the ICU.

Secondary outcomes include the following:

1. ICU mortality, 28-day mortality.

2. Changes in SOFA scores.

3. Changes in plasma biomarkers of inflammation: including white cell counts, neutrophil counts/percentage, lymphocyte counts/percentage, PCT, CRP and cytokines (IL-6, IL-10 and so on).

4. Changes in pulmonary infection estimated by chest radiography/CT imaging and lung ultrasound and changes in pulmonary function measured by Murray lung injury scores.

5. Rate of organ failure including ARDS diagnosed by the Berlin definition, ${ }^{53}$ AKI diagnosed by the Kidney Disease Improving Global Outcomes criteria,${ }^{54}$ coagulation disorders, immune dysfunction characterised by changes in lymphocytes and so on.

6. Other clinical outcomes including ventilation days, vasopressor days, ICU length of stay and hospital length of stay to day 28.

\section{SAMPLE SIZE AND INTERIM ANALYSIS}

Since the COVID-19 pandemic is uncertain and unpredictable, the sample size is calculated in two stages, which is set to adjust statistical power and determine whether there is a promising trend. At the first stage, as no data from COVID-19 regarding our primary outcome are available, ICU mortality $(13.57 \%$ reported by the WHO on 12 February $^{55}$ ) was chosen instead for the calculation of sample size through a non-inferiority test to maximise the possibility of significant results. With an allowable error of $10 \%$, a total of 308 study participants (154 in each group) would result in a power of $80 \%$ with a one-sided type 1 error rate $(\alpha)$ of $2.5 \%$, allowing a $10 \%$ withdrawal rate in each group. Of course, this calculated sample size is not accurate and needs revision based on the real situation. Thus, we arranged a second-stage calculation. After 50 patients are enrolled and have completed follow-up, interim analysis will be conducted at the second stage, and the required sample size will be recalculated. During interim analysis, the data of these 50 patients will be used and processed according to the following methods. The results of the analysis will be reported to the DSMB.

\section{STATISTICAL ANALYSIS}

Measurement data will be described as the mean with SD or median with IQR according to its distribution. Count data will be represented as frequencies and proportions. For univariate analysis, the difference in measurement data will be compared between the $\mathrm{VC}$ and placebo groups using t-test/Mann-Whitney $\mathrm{U}$ test. $\chi^{2}$ test and Fisher's exact test will be used for rate comparisons. A mixed linear model will be used to assess the effect of treatment on outcomes and to fit a repeated-measures analysis of variance. Kaplan-Meier analysis with the Wilcoxon test will be used to show the effect of VC on patient survival probability. The testing will be two sided, and a $\mathrm{p}$ value $<0.05$ will be considered statistically significant. SPSS V.22.0 and GraphPad Prism V.6.00 will be used to complete data processing and statistical analysis.

\section{PRIMARY AND SECONDARY ANALYSIS}

To describe the patients' baseline characteristics, ensure no differences in distribution and eliminate the confounding factors, the primary analysis will be an intention-to-treat comparison of the general characteristics (sex, age, race, group assignment, principle diagnosis and so on) and other random effects. The secondary analysis will be a comparison of the primary outcome and secondary outcomes between the treatment group and the control group. The statistical methods mentioned above will be applied according to the characteristics and distribution of the data.

\section{ADDITIONAL ANALYSIS}

If the number of enrolled patients permits (no fewer than five patients in each subgroup of interest), stratified analyses will be carried out in the following subgroups to further clarify the suitable population for administering HIVC:

1. Patients without ventilation and patients with mechanical ventilation or with a ventilation requirement within 24 hours after admission to the ICU.

2. Patients with high (above median) or low (below median) Murray lung injury scores at the time of randomisation.

3. Patients with high (above median) or low (below median) APACHE II scores at the time of randomisation.

4. Patients with different severities of ARDS, stratified into mild, moderate and severe groups based on RIF.

5. Patients receiving or not receiving renal replacement therapy. 


\section{HANDLING OF MISSING DATA}

For every patient, the days of mechanical ventilation and ventilator parameters will be recorded in the medical record system. Thus, data on the primary outcome is very unlikely to be missing. Secondary outcome data might be missing due to record-keeping errors. Multiple imputation methods, including mean completer, hot-deck imputation and regression, will be used to minimise the effect of missing data as much as possible. The data of patients with complete outcomes after conservative imputation will be included in the comparison between the treatment and control groups.

\section{DATA SAFETY MONITORING}

An independent DSMB comprised of two academic intensivists outside the study who are experienced in conducting clinical trials in critical illness is monitoring the progress and safety of the trial. The DSMB is able to pause the trial to investigate or give suggestions on potential safety issues to improve the study design and implementation.

\section{DISCUSSION}

Previous reports revealed that cytokine storms, which can be suppressed by VC, are believed to be the main mechanism in the deterioration of patients with COVID-19.911-13 $\mathrm{VC}$, traditionally considered a dietary supplement, has antimicrobial and immunomodulatory properties. HIVC has been proven to be safe and therapeutic in critical care medicine, primarily as an adjunct to the treatment of septic shock and multiple organ failure, where it has been shown to improve outcomes and reduce mortality. ${ }^{15}$ 36-39 'High-dose' VC therapy lacks a universal definition. A previous meta-analysis ${ }^{56}$ considered high doses as equal to or greater than $10 \mathrm{~g} /$ day. Twenty-four grams of $\mathrm{VC}$ in this trial is higher than the dosage of $\mathrm{VC}$ in previous clinical trials conducted on critically ill patients with severe infection. ${ }^{3738}$

To our knowledge, this is one of the first prospective randomised controlled studies of high-dose intravenous VC treatment for COVID-19. Specific treatments for COVID-19 are not available at present. There are several clinical trials exploring immunotherapy. ${ }^{10}$ Documenting efficacy for any of these approaches requires time, adequate patient numbers and careful analysis. Vaccine development will require even more time for both safety and efficacy testing. Although blood plasma treatment for critical patients with COVID-19 was recommended by the National and Health Commission of the People's Republic of China, plasma from recovered patients is a rare resource. Compared with these therapies, HIVC has great advantages in terms of stability, availability, safety and cost. Therefore, this trial is highly meaningful as it could potentially save lives at a low price. HIVC is expected to improve pulmonary function and reduce mortality for patients with COVID-19. Furthermore, it may also provide a basis and reference for HIVC treatment of other diseases with similar mechanisms.

However, there are also limitations to our study design and uncertainties during implementation. First, as SARS-CoV-2 is a novel coronavirus, the duration and distribution of infected cases are unpredictable geographically and temporally, so the number of recruited patients at some clinical centres may be low. Thus, competitive enrolment and multiple clinical trial centres are needed to ensure an adequate number of subjects. Second, complete blinding is not feasible due to the lack of available resources, such as placebos in the same package as VC. The study is unblinded for dosing nurses, attending physicians and investigators in charge of enrolling participants, but blinding will be maintained for patients and all other members of the clinical and research team, such as statistical staff, to minimise bias. Third, some patients may have received non-specific, tentative and explorative symptomatic treatment, such as interferon and traditional Chinese medicine, as there is no effective and standardised guideline for treating COVID-19 at the early stage, despite the National and Health Commission recommendations for the diagnosis and management of COVID-19, ${ }^{49}$ which continues to be updated. These therapies are expected to be effective, but they may act as confounding factors for the trial, and it must be taken into account for data analysis.

\section{TRIAL STATUS}

Patient recruitment started on 14 February 2020 and will be completed on 30 September 2020.

Acknowledgements This trial is conducted in two hospitals: Zhongnan Hospital of Wuhan University and Leishenshan Hospital of Wuhan City. The authors would like to thank the patients and their family members and the nurses, pharmacy staff, fellows and clinicians of the intensive care units at the above hospitals for making this study possible. We would also like to express our gratitude to the medical assistance teams for their help and sacrifice for Wuhan.

Contributors FL, YZ, JZ, YL, and ZP designed this study; FL, YZ, and JZ designed the statistical analysis plan; $J Z$ and $Y L$ wrote the bid for the research project and obtained permission from the ethics committees; FL, YZ, JZ, and YL carried out this trial; $F L$ and $Y Z$ drafted this manuscript; ZP, YL, and JZ carefully reviewed the manuscript; and all authors read and approved the final manuscript.

Funding This work was supported by the Science and Technology Department of Hubei Province (grant number: 2020FCA024) and the Special Project for Significant New Drug Research and Development in the Major National Science and Technology Projects of China (grant number: 2020ZX09201007). The foundations have reviewed and approved this project. The primary investigator is supposed to report the progress of this trial to the departments regularly.

Competing interests None declared.

Patient and public involvement Patients and/or the public were not involved in the design, or conduct, or reporting, or dissemination plans of this research.

Patient consent for publication Not required.

Ethics approval This study follows the principles of the Helsinki Declaration 2013. The entire protocol was reviewed and approved by the Ethics Committee of Zhongnan Hospital of Wuhan University (identifier: Clinical Ethical Approval No. 2020001). Zhongnan Hospital of Wuhan University also took charge of the management of Leishenshan Hospital of Wuhan City, including affairs of clinical trials, so the above ethical approval document is also applicable to Leishenshan Hospital. Written informed consent will be obtained from all participants who will be informed about the purpose, intervention and possible risks/benefits of the study. 
Provenance and peer review Not commissioned; externally peer reviewed.

Open access This is an open access article distributed in accordance with the Creative Commons Attribution Non Commercial (CC BY-NC 4.0) license, which permits others to distribute, remix, adapt, build upon this work non-commercially, and license their derivative works on different terms, provided the original work is properly cited, appropriate credit is given, any changes made indicated, and the use is non-commercial. See: http://creativecommons.org/licenses/by-nc/4.0/.

Author note The trial was registered on ClinicalTrials.gov (identifier: NCT04264533) on 4 February 2020. Of note, this manuscript is a revised version of the protocol that was reviewed and approved on 19 February 2020. The first version was approved on 31 January 2020. Compared with the first version, which was registered on ClinicalTrials.gov, the sample size and secondary outcomes of this version have been revised, as there was insufficient information about COVID-19 at the early stage, and we have learned more about COVID-19 over time.

ORCID iD

Zhiyong Peng http://orcid.org/0000-0002-0849-5648

\section{REFERENCES}

1 Coronavirus disease 2019 (COVID-19) situation report - 153: World Health organization, 2020. Available: https://www.who.int/ emergencies/diseases/novel-coronavirus-2019/situation-reports [Accessed 22 June 2020].

2 Huang C, Wang Y, Li X, et al. Clinical features of patients infected with 2019 novel coronavirus in Wuhan, China. The Lancet 2020;395:497-506.

3 Wang D, Hu B, Hu C, et al. Clinical characteristics of 138 hospitalized patients with 2019 novel coronavirus-infected pneumonia in Wuhan, China. JAMA 2020. doi:10.1001/jama.2020.1585. [Epub ahead of print: 07 Feb 2020].

4 Rodriguez-Morales AJ, Cardona-Ospina JA, Gutiérrez-Ocampo $\mathrm{E}$, et al. Clinical, laboratory and imaging features of COVID-19: a systematic review and meta-analysis. Travel Med Infect Dis 2020;34:101623.

5 Wang Y, Zhang D, Du G, et al. Remdesivir in adults with severe COVID-19: a randomised, double-blind, placebo-controlled, multicentre trial. Lancet 2020;395:1569-78.

6 Geleris J, Sun Y, Platt J, et al. Observational study of hydroxychloroquine in hospitalized patients with Covid-19. N Engl J Med 2020;382:2411-8.

7 Cao B, Wang Y, Wen D, et al. A trial of Lopinavir-Ritonavir in adults hospitalized with severe Covid-19. N Engl J Med 2020;382:1787-99.

8 Shimabukuro-Vornhagen A, Gödel P, Subklewe M, et al. Cytokine release syndrome. J Immunother Cancer 2018;6:56.

9 Channappanavar R, Perlman S. Pathogenic human coronavirus infections: causes and consequences of cytokine storm and immunopathology. Semin Immunopathol 2017;39:529-39.

10 Tu Y-F, Chien C-S, Yarmishyn AA, et al. A review of SARS-CoV-2 and the ongoing clinical trials. Int J Mol Sci 2020;21:2657.

11 Wan S, Yi Q, Fan S, et al. Characteristics of lymphocyte subsets and cytokines in peripheral blood of 123 hospitalized patients with 2019 novel coronavirus pneumonia (NCp). medRxiv 20202020.

12 Zhou Y, Fu B, Zheng X, et al. Aberrant pathogenic GM-CSF+ T cells and inflammatory CD14+ CD16+ monocytes in severe pulmonary syndrome patients of a new coronavirus. bioRxiv 2020.

13 Xu Z, Shi L, Wang Y, et al. Pathological findings of COVID-19 associated with acute respiratory distress syndrome. Lancet Respir Med 2020;8:420-2.

14 Mousavi S, Bereswill S, Heimesaat MM. Immunomodulatory and antimicrobial effects of vitamin C. Eur J Microbiol Immunol 2019;9:73-9.

15 Hemilä H, Chalker E. Vitamin $\mathrm{C}$ can shorten the length of stay in the ICU: a meta-analysis. Nutrients 2019;11. doi:10.3390/nu11040708. [Epub ahead of print: 27 Mar 2019].

16 Sauberlich HE, Packer L, Fuchs J. A history of scurvy and vitamin C. Vitamin C in Health \& Disease, 1997.

17 Carr AC, Maggini S. Vitamin C and immune function. Nutrients 2017;9. doi:10.3390/nu9111211. [Epub ahead of print: 03 Nov 2017].

18 Johnston CS, Beezhold BL, Mostow B, et al. Plasma vitamin C is inversely related to body mass index and waist circumference but not to plasma adiponectin in nonsmoking adults. $J$ Nutr 2007;137:1757-62.

19 Johnston CS, Corte C, Swan PD. Marginal vitamin C status is associated with reduced fat oxidation during submaximal exercise in young adults. Nutr Metab 2006;3:35.
20 Sen CK, Packer L. Antioxidant and redox regulation of gene transcription. Faseb J 1996;10:709-20.

21 Härtel C, Strunk T, Bucsky P, et al. Effects of vitamin C on intracytoplasmic cytokine production in human whole blood monocytes and lymphocytes. Cytokine 2004;27:101-6.

22 Chen Y, Luo G, Yuan J, et al. Vitamin C mitigates oxidative stress and tumor necrosis factor-alpha in severe community-acquired pneumonia and LPS-induced macrophages. Mediators Inflamm 2014;2014:426740

23 Cárcamo JM, Bórquez-Ojeda O, Golde DW. Vitamin C inhibits granulocyte macrophage-colony-stimulating factor-induced signaling pathways. Blood 2002;99:3205-12.

24 Maeng HG, Lim H, Jeong Y-J, et al. Vitamin C enters mouse T cells as dehydroascorbic acid in vitro and does not recapitulate in vivo vitamin C effects. Immunobiology 2009;214:311-20.

25 Gao Y-L, Lu B, Zhai J-H, et al. The parenteral vitamin C improves sepsis and sepsis-induced multiple organ dysfunction syndrome via preventing cellular immunosuppression. Mediators Inflamm 2017;2017:4024672

26 Tanaka M, Muto N, Gohda E, et al. Enhancement by ascorbic acid 2-glucoside or repeated additions of ascorbate of mitogen-induced IgM and IgG productions by human peripheral blood lymphocytes. Jpn J Pharmacol 1994;66:451-6.

27 Huijskens MJAJ, Walczak M, Sarkar S, et al. Ascorbic acid promotes proliferation of natural killer cell populations in culture systems applicable for natural killer cell therapy. Cytotherapy 2015;17:613-20.

28 Kratzer E, Tian $\mathrm{Y}$, Sarich N, et al. Oxidative stress contributes to lung injury and barrier dysfunction via microtubule destabilization. Am J Respir Cell Mol Biol 2012;47:688-97.

29 Delgado-Roche L, Mesta F. Oxidative stress as key player in severe acute respiratory syndrome coronavirus (SARS-CoV) infection. Arch Med Res 2020;51:384-7.

$30 \mathrm{Jin}$ X, Su R, Li R, et al. Amelioration of particulate matter-induced oxidative damage by vitamin $\mathrm{C}$ and quercetin in human bronchial epithelial cells. Chemosphere 2016;144:459-66.

31 Lang JD, McArdle PJ, O'Reilly PJ, et al. Oxidant-Antioxidant balance in acute lung injury. Chest 2002;122:314S-20.

32 Fisher BJ, Kraskauskas D, Martin EJ, et al. Mechanisms of attenuation of abdominal sepsis induced acute lung injury by ascorbic acid. Am J Physiol Lung Cell Mol Physiol 2012;303:L20-32.

33 Bharara A, Grossman C, Grinnan D, et al. Intravenous vitamin C administered as adjunctive therapy for recurrent acute respiratory distress syndrome. Case Rep Crit Care 2016;2016:1-4.

34 Furuya A, Uozaki M, Yamasaki H, et al. Antiviral effects of ascorbic and dehydroascorbic acids in vitro. Int J Mol Med 2008;22:541-5.

35 Padayatty SJ, Sun H, Wang Y, et al. Vitamin C pharmacokinetics: implications for oral and intravenous use. Ann Intern Med 2004;140:533.

36 Hemilä $\mathrm{H}$, Chalker $\mathrm{E}$. Vitamin $\mathrm{C}$ may reduce the duration of mechanical ventilation in critically ill patients: a meta-regression analysis. J Intensive Care 2020;8:15.

37 Fowler AA, Syed AA, Knowlson S, et al. Phase I safety trial of intravenous ascorbic acid in patients with severe sepsis. $J$ Trans Med 2014;12:32.

38 Fowler AA, Truwit JD, Hite RD, et al. Effect of vitamin C infusion on organ failure and biomarkers of inflammation and vascular injury in patients with sepsis and severe acute respiratory failure: the CITRISALI randomized clinical trial. JAMA 2019;322:1261.

39 Fowler lii AA, Kim C, Lepler L, et al. Intravenous vitamin C as adjunctive therapy for enterovirus/rhinovirus induced acute respiratory distress syndrome. World J Crit Care Med 2017:6:85-90.

40 Barness LA. Safety considerations with high ascorbic acid dosage. Ann N Y Acad Sci 1975;258:523-8.

41 Buehner M, Pamplin J, Studer L, et al. Oxalate nephropathy after continuous infusion of high-dose vitamin $\mathrm{C}$ as an adjunct to burn resuscitation. J Burn Care Res 2016;37:e374-9.

42 Campbell GD, Steinberg MH, Bower JD. Letter: ascorbic acidinduced hemolysis in G-6-PD deficiency. Ann Intern Med 1975;82:810.

43 Andres A, Zeier M, Eckert C. Acute kidney injury following high-dose vitamin C treatment. Dtsch Arztebl Int 2019;116:756.

44 Fijen L, Weijmer M. Acute oxalate nephropathy due to high vitamin $\mathrm{C}$ doses and exocrine pancreatic insufficiency. BMJ Case Rep 2019;12. doi:10.1136/bcr-2019-231504. [Epub ahead of print: 19 Nov 2019]

45 Padayatty SJ, Sun AY, Chen Q, et al. Vitamin C: intravenous use by complementary and alternative medicine practitioners and adverse effects. PLoS One 2010;5:e11414.

46 Khoshnam-Rad N, Khalili H. Safety of vitamin C in sepsis: a neglected topic. Curr Opin Crit Care 2019;25:329-33. 
47 Chan A-W, Tetzlaff JM, Altman DG, et al. Spirit 2013 statement: defining standard protocol items for clinical trials. Ann Intern Med 2013;158:200-7.

48 Ware $\mathrm{JH}$, Hamel MB. Pragmatic trials--guides to better patient care? N Engl J Med 2011;364:1685-7.

49 The Guideline for COVID-19 (version 5) issued by the National health Commission of the people's Republic of China, 2020. Available: http://www.nhc.gov.cn/yzygj/s7653p/202002/3b09b894ac9b4204 a79db5b8912d4440.shtml [Accessed 5 Feb 2020].

50 Wilson MK, Baguley BC, Wall C, et al. Review of high-dose intravenous vitamin $\mathrm{C}$ as an anticancer agent. Asia Pac J Clin Oncol 2014;10:22-37.

51 The good clinical practice (GCP) issued by the National medical administration of the people's Republic of China, 2003. Available: http://www.gov.cn/gongbao/content/2004/content_63115.htm [Accessed 6 Aug 2003].
52 Riordan HD, Hunninghake RB, Riordan NH, et al. Intravenous ascorbic acid: protocol for its application and use. P R Health Sci J 2003;22:287-90.

53 , Ranieri VM, Rubenfeld GD, et al, ARDS Definition Task Force. Acute respiratory distress syndrome: the Berlin definition. JAMA 2012;307:2526-33.

54 Khwaja A. KDIGO clinical practice guidelines for acute kidney injury. Nephron Clin Pract 2012;120:c179-84.

55 Coronavirus disease 2019 (COVID-19) situation report - 23: World Health organization, 2020. Available: https://www.who.int/ emergencies/diseases/novel-coronavirus-2019/situation-reports [Accessed 12 Feb 2020].

56 Wang Y, Lin H, Lin B-W, et al. Effects of different ascorbic acid doses on the mortality of critically ill patients: a meta-analysis. Ann Intensive Care 2019;9:58. 